\title{
On a Property of a Three-Dimensional Matrix
}

\author{
David Blokh \\ C. D. Technologies Ltd., Israel \\ Correspondence should be addressed to David Blokh; david_blokh@012.net.il
}

Received 11 June 2013; Accepted 24 September 2013

Academic Editor: Hong J. Lai

Copyright (C) 2013 David Blokh. This is an open access article distributed under the Creative Commons Attribution License, which permits unrestricted use, distribution, and reproduction in any medium, provided the original work is properly cited.

Let $S_{n}$ be the symmetrical group acting on the set $\{1,2, \ldots, n\}$ and $x, y \in S_{n}$. Consider the set $W=\{(i, x(i), y(i)) \mid 1 \leq i \leq n$, $|i-x(i)|>1 \vee|i-y(i)|>1 \vee|x(i)-y(i)|>1\}$. The main result of this paper is the following theorem. If the number of $W$ set entries is more than $[n / 3]$, then there exist entries $\left(i_{1}, x\left(i_{1}\right), y\left(i_{1}\right)\right),\left(i_{2}, x\left(i_{2}\right), y\left(i_{2}\right)\right),\left(i_{3}, x\left(i_{3}\right), y\left(i_{3}\right)\right) \in W$ such that $\left|i_{1}-x\left(i_{2}\right)\right| \leq 1$, $\left|i_{1}-y\left(i_{3}\right)\right| \leq 1$, and $\left|x\left(i_{2}\right)-y\left(i_{3}\right)\right| \leq 1$. The application of this theorem to the three-dimensional assignment problem is considered.

\section{Introduction}

Let $R_{n}^{3}$ be the set of $n \times n \times n$-matrices over the field of real numbers.

Three-dimensional matrix not only is an interesting mathematical object [1-3], but also has applications in many fields, such as theoretical physics [4] and operational research $[5,6]$.

Let $S_{n}$ be the symmetrical group acting on the set $\{1,2, \ldots, n\}, x, y \in S_{n},\left\|a_{i j k}\right\| \in R_{n}^{3}$ and

$$
\begin{gathered}
G=\left\{a_{i j k}|| i-j|\leq 1 \wedge| i-k|\leq 1 \wedge| j-k \mid \leq 1\right\}, \\
H=\left\{a_{i j k}|| i-j|>1 \vee| i-k|>1 \vee| j-k \mid>1\right\}, \\
T=\left\{a_{i x(i) y(i)} \mid 1 \leq i \leq n\right\} .
\end{gathered}
$$

The main result of this paper is the following theorem.

Theorem 1. If $\left\|a_{i j k}\right\| \in R_{n}^{3}, x, y \in S_{n}$ and the number of $T \cap$ $H$ set entries is more than $[n / 3]$, then there exist entries $a_{i_{1} j_{1} k_{1}}$, $a_{i_{2} j_{2} k_{2}}$, and $a_{i_{3} j_{3} k_{3}} \in T \cap H$ such that the entry $a_{i_{1} j_{2} k_{3}} \in G$.

We give another formulation of Theorem 1.

Consider the set

$$
\begin{aligned}
W=\{(i, x(i), y(i))|1 \leq i \leq n,| i-x(i) \mid \\
>1 \vee|i-y(i)|>1 \vee|x(i)-y(i)|>1\} .
\end{aligned}
$$

Theorem 2. If the number of $W$ set entries is more than $[n / 3]$, then there exist entries $\left(i_{1}, x\left(i_{1}\right), y\left(i_{1}\right)\right),\left(i_{2}, x\left(i_{2}\right), y\left(i_{2}\right)\right)$, $\left(i_{3}, x\left(i_{3}\right), y\left(i_{3}\right)\right) \in W$ such that $\left|i_{1}-x\left(i_{2}\right)\right| \leq 1,\left|i_{1}-y\left(i_{3}\right)\right| \leq 1$, and $\left|x\left(i_{2}\right)-y\left(i_{3}\right)\right| \leq 1$.

\section{Proof of Theorem 1}

We prove the theorem by contradiction. The set of matrix $\left\|a_{i j k}\right\| \in R_{n}^{3}$ entries with one index fixed and the two others having values from 1 to $n$ will be called a layer. We denote a layer by $L_{s}^{r}$, where $r$ indicates the location of a fixed index and $s$ indicates its value. For example, $L_{s}^{2}=\left\{a_{i s k} \mid i, k=1, \ldots, n\right\}$. Furthermore, entries from $G$ will be called basic, entries from $H$ will be called nonbasic; $T$ will be termed a trajectory; the layer containing a basic trajectory entry will be termed a basic layer and the layer containing a nonbasic trajectory entrywill be termed a nonbasic layer.

If $L_{s}^{1}$ is a nonbasic layer, then one layer in the pair $L_{s-1}^{2}, L_{s-1}^{3}$ is basic.

Suppose to the contrary that layers $L_{s}^{1}, L_{s-1}^{2}$, and $L_{s-1}^{3}$ are nonbasic. Let $a_{s j_{1} k_{1}}, a_{i_{2}, s-1, k_{2}}$, and $a_{i_{3}, j_{3}, s-1}$ be the trajectory entries of $L_{s}^{1}, L_{s-1}^{2}, L_{s-1}^{3}$ layers. Replace $a_{s j_{1} k_{1}}, a_{i_{2}, s-1, k_{2}}$, and $a_{i_{3}, j_{3}, s-1}$ with $a_{s, s-1, s-1}, a_{i_{2} j_{1} k_{1}}$, and $a_{i_{3} j_{3} k_{2}}$. The nonbasic trajectory entries $a_{s j_{1} k_{1}}, a_{i_{2}, s-1, k_{2}}$, and $a_{i_{3}, j_{3}, s-1}$ are replaced with entries $a_{s, s-1, s-1}, a_{i_{2} j_{1} k_{1}}$, and $a_{i_{3} j_{3} k_{2}}$, among which there is a basic one.

Similar assertions may be proved for the $L_{s}^{1}$ layer and the following layer pairs: $L_{s-1}^{2}, L_{s}^{3} ; L_{s}^{2}, L_{s-1}^{3} ; L_{s}^{2}, L_{s}^{3} ; L_{s}^{2}, L_{s+1}^{3}$; $L_{s+1}^{2}, L_{s}^{3}$; and $L_{s+1}^{2}, L_{s+1}^{3}$.

Three nonbasic layers may not be consecutive. 
Suppose that layers $L_{s-1}^{1}, L_{s}^{1}$, and $L_{s+1}^{1}$ are nonbasic. One of the layers $L_{s}^{2}$ or $L_{s}^{3}$ is basic. In these layers, the basic entries may be $a_{s-1, s, s-1}, a_{s-1, s, s}, a_{s, s, s-1}, a_{s s s}, a_{s, s, s+1}, a_{s+1, s, s}$, and $a_{s+1, s, s+1}$ for the layer $L_{s}^{2}$, and $a_{s-1, s-1, s}, a_{s-1, s, s}, a_{s, s-1, s}$, $a_{s s s}, a_{s+1, s, s}, a_{s, s+1, s}, a_{s+1, s+1, s}$ for the layer $L_{s}^{3}$. However, this contradicts the assumption that the layers $L_{s-1}^{1}, L_{s}^{1}$, and $L_{s+1}^{1}$ are nonbasic. The fact that the two first and the two last layers may not be nonbasic is proved in a similar way.

All assertions given below represent conditions that prevent replacing nonbasic trajectory entries with entries that include a basic one.

Consider the sequence of layers $L_{1}^{1}, L_{2}^{1}, \ldots, L_{s-1}^{1}, L_{s}^{1}$, $L_{s+1}^{1}, \ldots, L_{n-1}^{1}$, and $L_{n}^{1}$. Given below are possible arrangements of layers in this sequence. A basic layer $L_{s}^{1}$ is denoted by $\mathbf{1}$; a nonbasic layer $L_{s}^{1}$ is denoted by $\mathbf{0}$.

Consider

(1) ..11100111. . ; 1100111. . ; . . 1110011; 110011.

(2) ..111010111...; 01111010 . . ..01011110; ..001111010 $\ldots ; \ldots 010111100 \ldots ; \ldots 0101111010 \ldots$

(3) $0111100 \ldots ; \ldots 00111100 \ldots ; \ldots 0011110$.

(4) $0111 . .$. ; . .1110; 10111... . . 11101; 011110.

We prove the first arrangement of item 1. Suppose that the two nonbasic layers are followed only by two basic layers, that is, ..00110... Let there be layers ... $L_{s}^{1}, L_{s+1}^{1}, L_{s+2}^{1}, L_{s+3}^{1}$, $L_{s+4}^{1} \cdots$..

Consider layer pairs $L_{s+1}^{2}, L_{s+1}^{3} ; L_{s+1}^{2}, L_{s+2}^{3} ; L_{s+2}^{2}, L_{s+1}^{3} ; L_{s+2}^{2}$, and $L_{s+2}^{3}$. At least one layer of each pair is basic. These four pairs of layers contain at least two basic entries, and these entries are located in layers $L_{s+2}^{1}$ and $L_{s+3}^{1}$. The pair $L_{s+3}^{2}, L_{s+3}^{3}$ includes a basic layer. The first coordinate of the basic layer entry may be $s+2, s+3$, or $s+4$. But the $L_{s+2}^{1}$ and $L_{s+3}^{1}$ layers are occupied, while the $L_{s+4}^{1}$ layer is nonbasic. There is a contradiction.

The other arrangements are proved in a similar way.

Thus, nonbasic layers may not be arranged closer than those in the above variants. But these variants do not allow for the composition of a combination containing more than $[n / 3]$ nonbasic layers. Hence, it follows that if a trajectory includes more than $[n / 3]$ nonbasic entries, then one of the variants is violated and nonbasic trajectory entries can be replaced by a set of entries containing a basic entry.

\section{Application to the Three-Dimensional Assignment Problem}

The three-dimensional assignment problem (AP3) is an important combinatorial optimization problem. It is sufficient to note that the particular case of AP3, the 3dimensional matching problem, is one of the six main NPhard problems [7]. The formal AP3 statement is as follows: for a matrix $\left\|a_{i j k}\right\| \in R_{n}^{3}$, find permutations $x, y \in S_{n}$ such that $\sum_{i=1}^{n} a_{i x(i) y(i)}$ is maximized.
In this paper, the AP3 is considered for a special class of $n \times n \times n$-matrices $U(n)$. A matrix

$$
\begin{aligned}
& \left\|a_{i j k}\right\| \in U(n), \quad \text { if } a_{i j k} \geq 0, \\
& \min _{a_{i j k} \in G} a_{i j k} \geq C \cdot \max _{a_{i j k} \in H} a_{i j k}, \quad C \geq 3 .
\end{aligned}
$$

One of AP3 interpretations is the following. There are $n$ employees and two job sets of $n$ jobs each. If the $i$ th employee performs the $j$ th job of the first set and the $k$ th job of the second set, then the effect equals $a_{i j k}$. It is required to distribute the jobs among the employees in such a way so as to maximize the total effect.

Let us describe the situation that will lead to the AP3 for matrices from $U(n)$. As a rule, the employees are ordered by qualification, while the jobs are ordered by complexity. A higher effect is reached when a more qualified employee performs a more complex job, and we may arrive at the AP3 for matrices from $U(n)$.

The particular cases of AP3 [5, 8-11], the nonpolynomial exact algorithms for the AP3 $[6,12]$, and heuristics for the AP3 $[13,14]$ were considered.

The NP-hard particular cases of the traveling salesman problem, with sets of matrices whose structures are similar to those of matrices from $U(n)$, have been considered previously $[15,16]$.

Theorem 3. The AP3 for matrices from $U(n)$ is NP-hard.

Proof. Let $\left\|b_{i j k}\right\| \in R_{n}^{3}, b_{i j k} \geq 0$ and numbers $A, B$ are such that $A=3 \cdot \max b_{i j k}, B=n A$.

Consider $4 n \times 4 n \times 4 n$-matrix $\left\|a_{i j k}\right\|$ with nonnegative entries

$$
\begin{gathered}
a_{4 s-3,4 s-2,4 s-2}=B \quad s=1, \ldots, n ; \\
a_{4 s-2,4 s-1,4 s-1}=B \quad s=1, \ldots, n ; \\
a_{4 s, 4 s, 4 s}=B \quad s=1, \ldots, n ; \\
a_{r u v}=A \quad r, u, v=1, \ldots, n ; \quad \text { if }|r-u| \leq 1, \\
|r-v| \leq 1, \quad|u-v| \leq 1, \quad a_{r u v} \neq B ; \\
a_{4 i-1,4 j-3,4 k-3}=b_{i j k} \quad i, j, k=1, \ldots, n ; \\
a_{i j k}=0 \text { in other cases. }
\end{gathered}
$$

The matrix $\left\|a_{i j k}\right\| \in U(4 n)$.

All entries that are equal to $B$ belong to the optimal solution of AP3 for $\left\|a_{i j k}\right\|$ matrix. This signifies that if the optimal solution of AP3 for $\left\|a_{i j k}\right\|$ matrix is known, then the optimal solution of AP3 for $\left\|b_{i j k}\right\|$ matrix is known as well. Therefore, the AP3 for arbitrary matrices is polynomially reducible to the AP3 for matrices from $U(n)$.

Corollary 4. If $\left\|a_{i j k}\right\| \in U(n)$, then a set T exists on which the AP3 optimum is reached and the number of $T \cap H$ set entries is no more than $[n / 3]$.

Indeed, if for optimal T of matrix $\left\|a_{i j k}\right\| \in U(n)$ the number of $T \cap H$ set entries is more than $[n / 3]$, then, according to 
Theorem 1, it is possible to make such replacement of entries such that the sum of $T$ set entries will not change and the number of $T \cap H$ set entries is reduced.

Theorem 5. If $\left\|a_{i j k}\right\| \in U(n)$, then the AP3 optimum for a $\left\|a_{i j k}^{\prime}\right\|$ matrix, where $a_{i j k}^{\prime}=a_{i j k}$ for $a_{i j k}^{\prime} \in G$ and $a_{i j k}^{\prime}=0$ for $a_{i j k}^{\prime} \in H$, represents an approximation for the AP3 optimum of the $\left\|a_{i j k}\right\|$ matrix with a relative error not exceeding $1 /(3 C)$.

Proof. Let $\left\|a_{i j k}\right\| \in U(n) ; m_{1}$ is the AP3 optimum for $\left\|a_{i j k}\right\|$; $f, g \in S_{n}$ are such that $m_{1}=\sum_{i=1}^{n} a_{i f(i) g(i)}$, and the number of $\left\{a_{i f(i) g(i)} \mid i=1, \ldots, n\right\} \cap H$ set entries is no more than $[n / 3]$; $m_{2}$ is the sum of $\left\{a_{i f(i) g(i)} \mid i=1, \ldots, n\right\} \cap H$ set entries and $m_{3}=m_{1}-m_{2} ; m^{\prime}$ is the AP3 optimum for $\left\|a_{i j k}^{\prime}\right\|$.

Note that $m_{3}=\sum_{i=1}^{n} a_{i f(i) g(i)}^{\prime}$ and $m^{\prime} \geq m_{3}$.

Insofar as $a_{i j k} \geq a_{i j k}^{\prime}, i, j, k=1, \ldots, n$, then $m_{1} \geq m^{\prime}$. Hence, $m_{3} \leq m^{\prime} \leq m_{1}$ and $\left(m_{1}-m^{\prime}\right) / m_{1} \leq\left(m_{1}-m_{3}\right) / m_{1}=$ $m_{2} / m_{1}$.

Since $m_{2} \leq(1 / 3) n \cdot \max _{a_{i j k} \in H} a_{i j k}$ and $m_{1} \geq n \cdot \min _{a_{i j k} \in G} a_{i j k}$, then $m_{2} / m_{1} \leq 1 /(3 C)$. Hence, $\left(m_{1}-m^{\prime}\right) / m_{1} \leq 1 /(3 C)$, and the relative error of $m^{\prime}$ taken as the AP3 approximate solution for the $\left\|a_{i j k}\right\|$ matrix is no more than $1 /(3 C)$.

Remark 6. An exact $O(n)$-algorithm of AP3 solution has been constructed [9] for $n \times n \times n$-matrices $\left\|a_{i j k}\right\|$ such that $a_{i j k} \geq$ 0 for $a_{i j k} \in G$ and $a_{i j k}=0$ for $a_{i j k} \in H$ using dynamical programming.

\section{Acknowledgments}

The author is grateful to Professor Y. Dinitz and Professor G. Gutin for their attention to this work and valuable comments. Also the author is grateful to the anonymous referee of this journal for the valuable comments and suggestions.

\section{References}

[1] R. A. Brualdi and J. Csima, "On the plane term rank of a three dimensional matrix," Proceedings of the American Mathematical Society, vol. 54, no. 1, pp. 471-473, 1976.

[2] J. Csima, "On the plane term rank of three dimensional matrices," Discrete Mathematics, vol. 28, no. 2, pp. 147-152, 1979.

[3] D. K. Kim, Y. A. Kim, and K. Park, "Generalizations of suffix arrays to multi-dimensional matrices," Theoretical Computer Science, vol. 302, no. 1-3, pp. 401-416, 2003.

[4] Y. Kawamura, "Cubic matrices, generalized spin algebra and uncertainty relation," Progress of Theoretical Physics, vol. 110, no. 3, pp. 579-587, 2003.

[5] R. E. Burkard, R. Rudolf, and G. J. Woeginger, “Three-dimensional axial assignment problems with decomposable cost coefficients," Discrete Applied Mathematics, vol. 65, no. 1-3, pp. 123139, 1996.

[6] W. P. Pierskalla, "The multidimensional assignment problem," Operations Research, vol. 16, no. 2, pp. 422-431, 1968.

[7] M. R. Garey and D. C. Johnson, Computer and Intractability: A Guide To the Theory of NP-Completeness, W. H. Freeman, San Francisco, Calif, USA, 1979.
[8] W. W. Bein, P. Brucker, J. K. Park, and P. K. Pathak, "A Monge property for the d-dimensional transportation problem," Discrete Applied Mathematics, vol. 58, no. 2, pp. 97-109, 1995.

[9] D. A. Blokh, "Assignment problem for three-index Jacobi matrices," Automation and Remote Control, vol. 50, no. 5, pp. 672-677, 1989.

[10] Y. Crama and F. C. R. Spieksma, "Approximation algorithms for three-dimensional assignment problems with triangle inequalities," European Journal of Operational Research, vol. 60, no. 3, pp. 273-279, 1992.

[11] F. C. R. Spieksma and G. J. Woeginger, "Geometric threedimensional assignment problems," European Journal of Operational Research, vol. 91, no. 3, pp. 611-618, 1996.

[12] E. Balas and M. J. Saltzman, "An algorithm for the three-index assignment problem," Operations Research, vol. 39, no. 1, pp. 150-161, 1991.

[13] G. Gutin, B. Goldengorin, and J. Huang, "Worst case analysis of Max-Regret, greedy and other heuristics for multidimensional assignment and traveling salesman problems," Journal of Heuristics, vol. 14, no. 2, pp. 169-181, 2008.

[14] B.-J. Kim, W. L. Hightower, P. M. Hahn, Y.-R. Zhu, and L. Sun, "Lower bounds for the axial three-index assignment problem," European Journal of Operational Research, vol. 202, no. 3, pp. 654-668, 2010.

[15] D. Blokh and G. Gutin, "Maximizing traveling salesman problem for special matrices," Discrete Applied Mathematics, vol. 56, no. 1, pp. 83-86, 1995.

[16] D. Blokh and E. Levner, "An approximation algorithm with performance guarantees for the maximum traveling salesman problem on special matrices," Discrete Applied Mathematics, vol. 119, no. 1-2, pp. 139-148, 2002. 


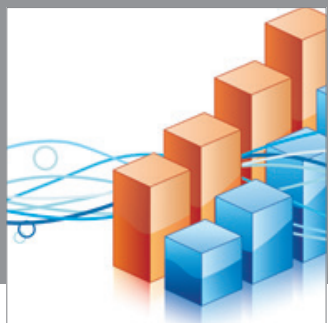

Advances in

Operations Research

mansans

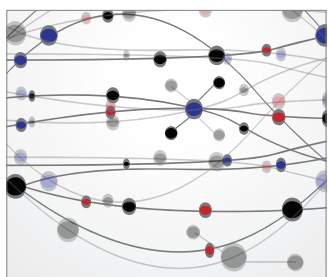

The Scientific World Journal
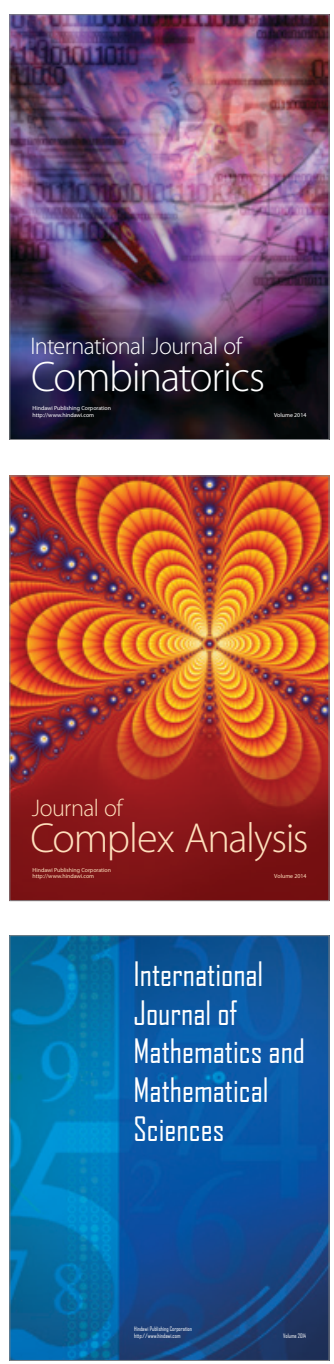
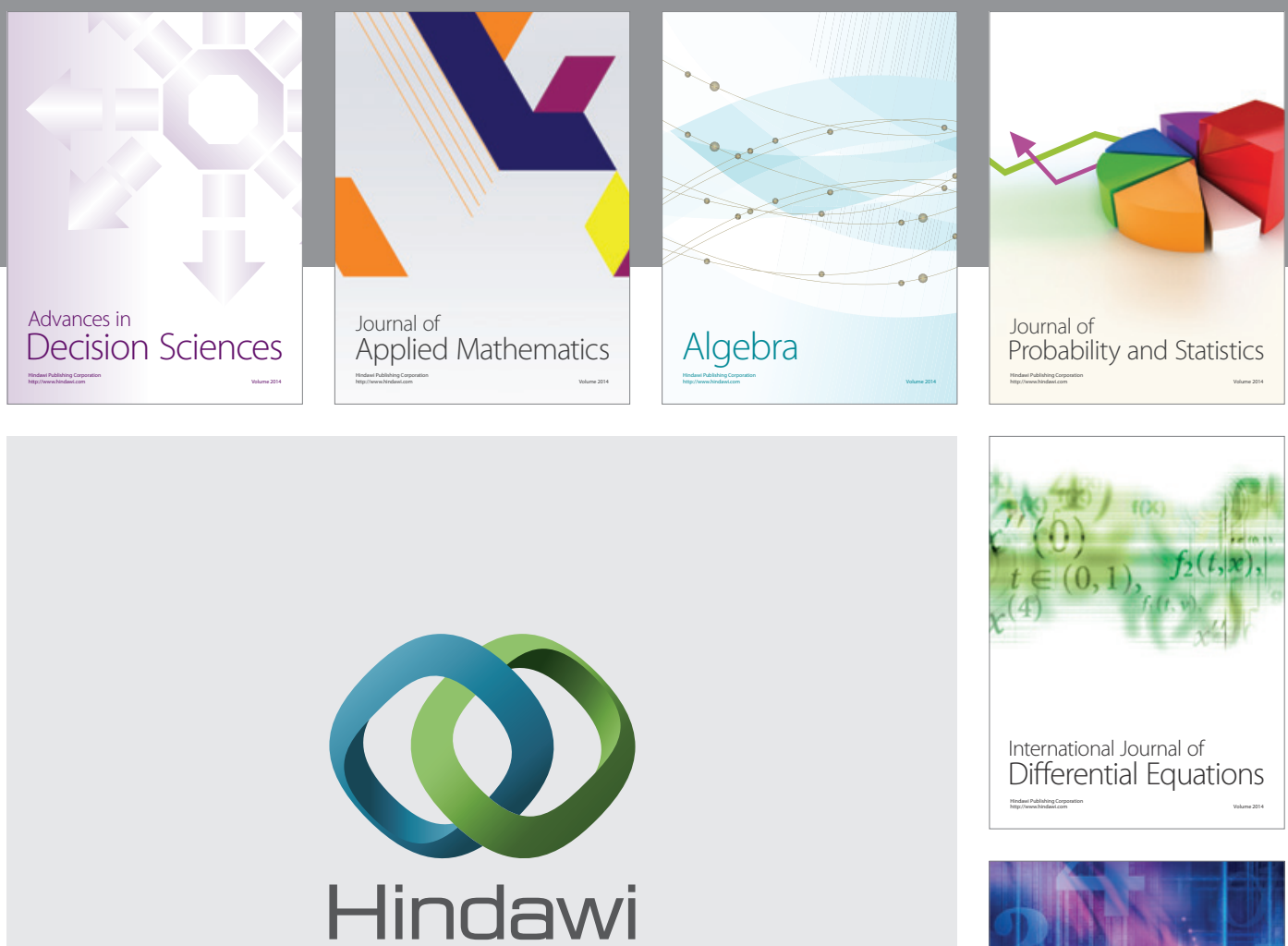

Submit your manuscripts at http://www.hindawi.com
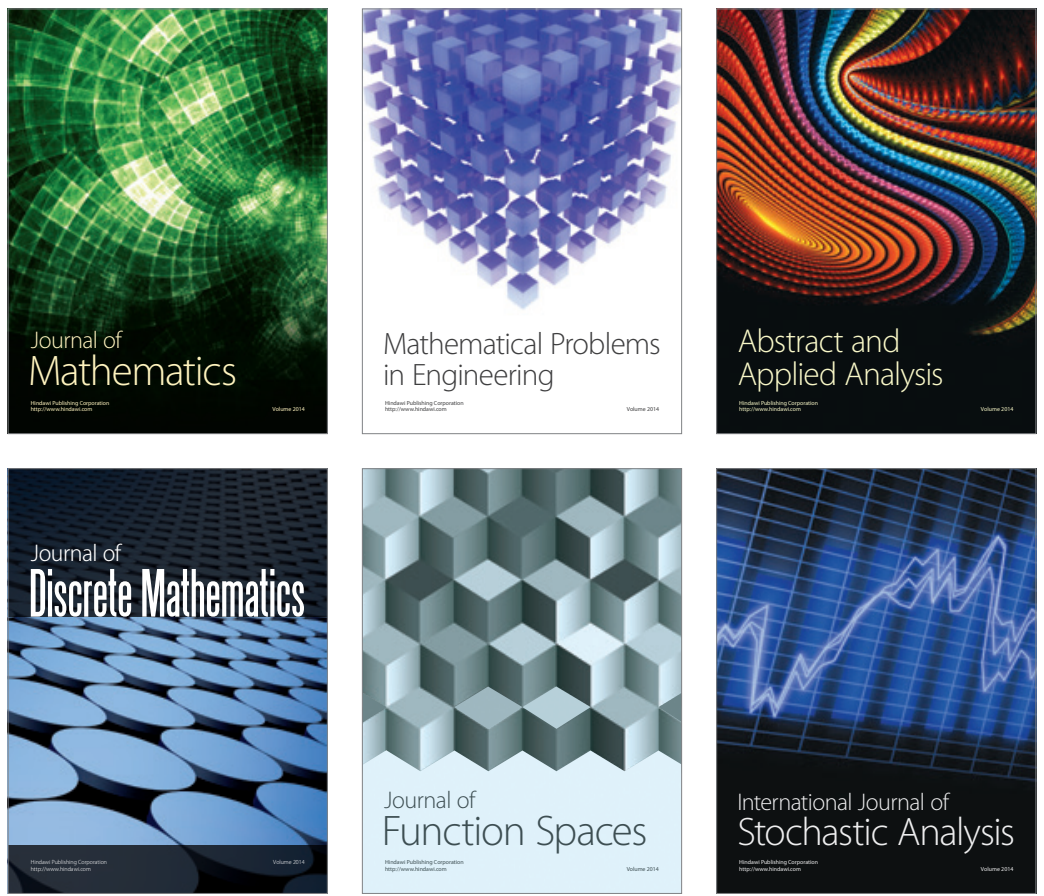

Journal of

Function Spaces

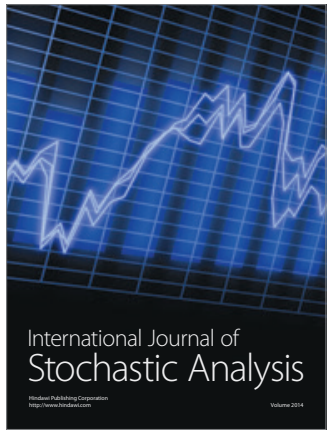

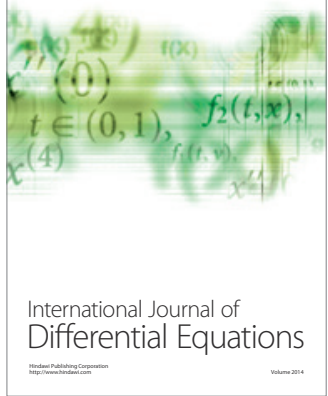
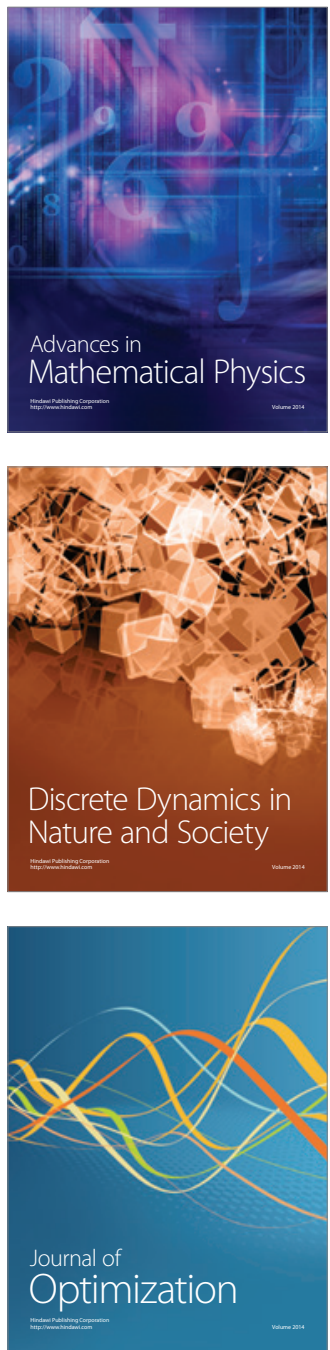